\title{
Inducible liver-specific knockdown of protein tyrosine phosphatase 1B improves glucose and lipid homeostasis in adult mice
}

\author{
C. Owen • E. K. Lees - L. Grant • D. J. Zimmer • \\ N. Mody $•$ K. K. Bence $\cdot$ M. Delibegović
}

Received: 29 May 2013 / Accepted: 14 June 2013 / Published online: 6 July 2013

(C) Springer-Verlag Berlin Heidelberg 2013

\begin{abstract}
Aims/hypothesis Protein tyrosine phosphatase 1B (PTP1B) is a key negative regulator of insulin signalling. Hepatic PTP1B deficiency, using the Alb-Cre promoter to drive Ptp $1 b$ deletion from birth in mice, improves glucose homeostasis, insulin sensitivity and lipid metabolism. The aim of this study was to investigate the therapeutic potential of decreasing liver PTP1B levels in obese and insulinresistant adult mice.

Methods Inducible Ptp1b liver-specific knockout mice were generated using $S A-C r e-E R^{T 2}$ mice crossed with Ptplb floxed $\left(P t p 1 b^{\mathrm{fl} / \mathrm{fl}}\right)$ mice. Mice were fed a high-fat diet (HFD) for 12 weeks to induce obesity and insulin resistance. Tamoxifen was administered in the HFD to induce liverspecific deletion of Ptp1b (SA-Ptp1 $b^{-/-}$mice). Body weight, glucose homeostasis, lipid homeostasis, serum adipokines, insulin signalling and endoplasmic reticulum (ER) stress were examined.

Results Despite no significant change in body weight relative to HFD-fed $P t p 1 b^{\mathrm{fl} / \mathrm{fl}}$ control mice, HFD-fed $S A$ $P t p 1 b^{-1-}$ mice exhibited a reversal of glucose intolerance as determined by improved glucose and pyruvate tolerance tests, decreased fed and fasting blood glucose and insulin levels, lower HOMA of insulin resistance, circulating leptin,
\end{abstract}

C. Owen and E. K. Lees contributed equally to this study.

C. Owen $\cdot$ E. K. Lees $\cdot$ L. Grant $\cdot$ N. Mody $\cdot$ M. Delibegović $(\square)$ Institute of Medical Sciences, School of Medical Sciences, University of Aberdeen, Foresterhill, Aberdeen AB25 2ZD, UK e-mail: m.delibegovic@abdn.ac.uk

\section{J. Zimmer $\cdot$ K. K. Bence $(\triangle)$}

Department of Animal Biology, School of Veterinary Medicine, University of Pennsylvania, 3800 Spruce Street, Vet 223E, Philadelphia, PA 19104-6046, USA

e-mail: kbence@vet.upenn.edu serum and liver triacylglycerols, serum NEFA and decreased HFD-induced ER stress. This was associated with decreased glycogen synthase, eukaryotic translation initiation factor$2 \alpha$ kinase 3, eukaryotic initiation factor $2 \alpha$ and c-Jun $\mathrm{NH}_{2}-$ terminal kinase 2 phosphorylation, and decreased expression of Pepck.

Conclusions/interpretation Inducible liver-specific PTP1B knockdown reverses glucose intolerance and improves lipid homeostasis in HFD-fed obese and insulin-resistant adult mice. This suggests that knockdown of liver PTP1B in individuals who are already obese/insulin resistant may have relatively rapid, beneficial therapeutic effects.

Keywords Disease · ER stress · Glucose · Insulin · Leptin · Lipid $\cdot$ Liver $\cdot$ NAFLD $\cdot$ Phosphatase $\cdot$ PTP1B

$\begin{array}{ll}\text { Abbreviations } \\ \text { ALT } & \text { Alanine aminotransferase } \\ \text { eIF2 } \alpha & \text { Eukaryotic initiation factor 2 } \alpha \\ \text { ER } & \text { Endoplasmic reticulum } \\ \text { ERK } & \text { Extracellular signal-regulated kinase } \\ \text { GS } & \text { Glycogen synthase } \\ \text { GSK-3 } & \text { Glycogen synthase kinase } 3 \\ \text { GTT } & \text { Glucose tolerance test } \\ \text { HFD } & \text { High-fat diet } \\ \text { HOMA-IR } & \text { HOMA of insulin resistance } \\ \text { IR } & \text { Insulin receptor } \\ \text { JAK2 } & \text { Janus kinase 2 } \\ \text { JNK } & \text { c-Jun NH } \text { H}_{2} \text {-terminal kinase } \\ \text { MCP-1 } & \text { Monocyte chemoattractant protein 1 } \\ \text { mTOR } & \text { Mammalian target of rapamycin } \\ \text { NAFLD } & \text { Non-alcoholic fatty liver disease } \\ \text { PERK } & \text { Eukaryotic translation initiation factor-2 } 2 \alpha \\ & \text { kinase } 3\end{array}$




$\begin{array}{ll}\text { PKB } & \text { Protein kinase B } \\ \text { PP2A } & \text { Protein phosphatase 2 } \\ \text { PTP1B } & \text { Protein tyrosine phosphatase 1B } \\ \text { PTT } & \text { Pyruvate tolerance test } \\ \text { SHP2 } & \text { SH2 domain-containing protein tyrosine } \\ & \text { phosphatase 2 } \\ \text { WAT } & \text { White adipose tissue }\end{array}$

\section{Introduction}

Caloric excess and low physical activity are key drivers of rising obesity levels in Western society. Insulin resistance and obesity are associated with the development of cardiovascular disease, type 2 diabetes and cancer [1]. Insulin resistance leads to hyperglycaemia, caused by a decrease in insulin-dependent glucose uptake into peripheral tissues and diminished ability of insulin to suppress hepatic glucose production [2]. Insulin resistance is also associated with dyslipidaemia [3] and nonalcoholic fatty liver disease (NAFLD), which is the most common liver disease throughout the world $(20-35 \%$ of the population) [4]. It is distinguished by excess hepatic fat stores, in the absence of alcohol consumption [4]. Lifestyle factors such as nutrition and exercise can influence whether NAFLD is likely to progress to non-alcoholic steatohepatitis [4], which carries an increased risk of mortality [5]. This rising burden of metabolic disease requires the development of new therapeutic strategies [6].

Protein tyrosine phosphatase 1B (PTP1B; also known as PTPN1) is a non-receptor tyrosine phosphatase which is ubiquitously expressed in all insulin-responsive tissues [7]. PTP1B is a negative regulator of both insulin and leptin signalling through its actions on the insulin receptor (IR) and Janus kinase 2 (JAK2) [7]. Whole-body Ptp1 $b^{-1-}$ mice have enhanced insulin sensitivity, increased phosphorylation of tyrosine residues on the IR and reduced adiposity on a high-fat diet (HFD) [8, 9]. PTP1B antisense oligonucleotides were shown to effectively lower PTP1B levels in liver and fat, enhance insulin signalling and decrease adiposity in $o b / o b$ and $d b / d b$ mice [10, 11]. Lipogenic genes were downregulated in fat and liver, including diminished Pparg expression in adipose tissue [12]. Whether these positive effects were due to loss of PTP1B in the liver and/or adipose tissue or any other tissue(s) was, however, unclear. Subsequently, tissue-specific Ptp $1 b$ knockout mice were generated to identify the specific tissues responsible for PTP1B's effects on insulin sensitivity and lipid metabolism [2, 13, 14].

On an HFD, neuronal $\mathrm{Ptp}_{1 b^{-/-}}$mice display decreased body mass, reduced food intake and enhanced energy expenditure [13]. This is due, at least in part, to leptin hypersensitivity in these mice; PTP1B acts as a negative regulator of leptin signalling via its ability to dephosphorylate JAK2 on tyrosine sites $\mathrm{Y} 1007 / \mathrm{Y} 1008$ and altering phosphorylation of downstream signal transducer and activator of transcription 3 [15]. Adipocyte-specific Ptp $1 b$ deletion increases adipocyte size and serum levels of glucose and leptin, without affecting body weight [6]. Muscle-specific and liver-specific deletion of Ptplb has no effect on body weight but, in contrast to adipocyte $P t p 1 b$ deletion, improves peripheral insulin sensitivity and whole-body glucose homeostasis $[2,14]$. Liverspecific Ptp $1 b$ deletion also decreases serum triacylglycerol levels and lowers lipogenic gene expression in livers of HFD-fed mice (Srebp1c, Srebp 1a and Fas). It is thought that this may be due to decreased endoplasmic reticulum (ER) stress response induction observed in these mice $[16,17]$.

Specifically targeting liver PTP1B appears to be an attractive drug therapy for the treatment of metabolic syndrome, as it not only improves whole-body insulin sensitivity and glucose homeostasis but also decreases lipid deposition in the liver, thus potentially limiting the development of comorbidities such as NAFLD.

Previous studies have examined the beneficial effects of liver PTP1B deficiency in Alb-Cre mice, which have hepatocyte Ptplb deletion from birth [2]. The aim of this study was to investigate whether inhibiting liver PTP1B in adult mice with established obesity and insulin resistance could reverse the phenotype and therefore present a novel treatment for insulin resistance and type 2 diabetes. To do this, we used a tamoxifen-dependent Cre recombinase system under the control of the serum albumin promoter to target PTP1B specifically in liver.

\section{Methods}

Ethics statement All animal procedures were approved by the University of Aberdeen Ethics Review Committee Board and performed under a project licence approved by the Home Office under the Animals (Scientific Procedures) Act 1986 (PPL60/3951).

Animal studies $P t p 1 b^{\mathrm{fl} / \mathrm{fl}}$ mice and $S A-C r e-E R^{T 2}$ mice expressing $C r e-E R^{T 2}$ recombinase under the control of the serum albumin promoter were described previously [18, 19]. Tamoxifen treatment of mice efficiently induces Cremediated recombination of LoxP flanked (floxed) alleles in hepatocytes but not in other cell types or tissues [19]. $S A$ Cre-ER ${ }^{T 2}$ mice, when crossed to $\operatorname{Ptp} 1 b^{\mathrm{fl} / \mathrm{fl}}$ mice, provide a tool to temporally control targeted mutagenesis in hepatocytes. Tamoxifen administration induces a liver-specific deletion of Ptplb (hereafter termed SA-Ptp $1 b^{-/-}$mice). DNA extraction and genotyping for the $P t p 1 b$ floxed allele and the presence of $C r e-E R^{T 2}$ by PCR were performed as described previously [18]. Mice studied were age-matched littermates, which were generated on a C57BL/6 background. Mice were 
housed in groups, unless otherwise stated, and maintained at $22-24^{\circ} \mathrm{C}$ on a $12 \mathrm{~h} \mathrm{light/dark} \mathrm{cycle} \mathrm{with} \mathrm{free} \mathrm{access} \mathrm{to} \mathrm{food}$ and water. At weaning ( 21 days), mice were placed on a standard 3.4\% fat chow pellet diet (Rat and Mouse Breeder and Grower; Special Diets Services, DBM, Bathgate, UK) or an HFD (Adjusted Calories Diet, 55\% fat; TD.93075, Harlan Teklad, Madison, WI, USA) and their weight was recorded every 2 weeks. The approximate fatty acid profile of the Adjusted Calories Diet (\% total fat) was $28 \%$ saturated, $30 \%$ trans, $28 \%$ monounsaturated (cis) and $14 \%$ polyunsaturated (cis), as described previously [20]. For insulin signalling experiments, HFD-fed mice were fasted overnight (16 h) and then injected i.p. with saline $(154 \mathrm{mmol} / \mathrm{l} \mathrm{NaCl})$ or insulin $(10 \mathrm{mU} / \mathrm{g}$ body weight $)$ for $10 \mathrm{~min}$. Tissues were dissected immediately after cervical dislocation and frozen in liquid nitrogen.

Tamoxifen administration To prepare tamoxifen, ethanol was added to make a $10 \mathrm{mg} / 100 \mu \mathrm{l}$ suspension. Sunflower seed oil was then added to prepare a $10 \mathrm{mg} / \mathrm{ml}$ solution. This was heated at $55^{\circ} \mathrm{C}$ for $30 \mathrm{~min}$. This mixture of tamoxifen, ethanol and sunflower oil was then incorporated into the HFD (55\% fat) at $0.7 \mathrm{mg}$ tamoxifen/g food. A control HFD (55\% fat) containing ethanol and sunflower oil only was simultaneously administered to a control group. Mice were fed the tamoxifen or control HFD for 28 days.

PTP1B activity assay Tissue lysates were prepared in PTP lysis buffer (130 mmol/l NaCl, 20 mmol/l TRIS (pH 7.5), $5 \mathrm{mmol} / \mathrm{l}$ EDTA, $1 \%$ Triton X-100 (v/v), 0.5\% Nonidet P-40 $(v / v)$-containing protease inhibitors). PTP1B protein was immunoprecipitated using PTP1B antibody (Millipore, Watford, UK) and protein G-sepharose beads. Beads were resuspended in $60 \mu \mathrm{l}$ of PTP assay buffer $(100 \mathrm{mmol} / \mathrm{l}$ HEPES [pH 7.6], 2 mmol/l EDTA, 1 mmol/l dithiothreitol (DTT), $150 \mathrm{mmol} / 1 \mathrm{NaCl}, 0.5 \mathrm{mg} / \mathrm{ml} \mathrm{BSA}$ ) containing phosphoregulatory peptide $(200 \mu \mathrm{mol} / \mathrm{l})$. The reaction proceeded for $30 \mathrm{~min}$ at $30^{\circ} \mathrm{C}$ with constant shaking. The concentration of phosphate produced $(\mu \mathrm{mol} / \mathrm{l})$ was then measured by absorbance at $620 \mathrm{~nm}$ using Biomol Green Reagent (Enzo Life Sciences, Exeter, UK) and phosphate standards.

Histology Frozen tissues were embedded in optimal cutting temperature compound (OCT) and sectioned by cryostat. Samples were stained in haematoxylin and eosin. Slides were visualised using a Zeiss Axioskop microscope (Carl Zeiss Microscopy, Thornwood, NY, USA) and imaged using AxioVision 4.8 digital image processing software (Carl Zeiss Microscopy).

Serum analysis Serum insulin and leptin (catalogue no. 90080 insulin/90030 leptin; CrystalChem, Downers Grove, IL, USA) were determined by ELISA, following manufacturer's instructions. TNF- $\alpha$, IL-6, monocyte chemoattractant protein 1 (MCP-1) and resistin were determined by multiplex ELISA assay (MADKMAG-71 K; Millipore), following manufacturer's instructions. Serum glucose (glucose oxidase, catalogue no. TR1503; Thermo Scientific, Cramlington, UK) and serum triacylglycerol (catalogue no. 17628; Sentinel Diagnostics, Milan, Italy; or catalogue no. TR0100; Sigma, Gillingham, UK) were determined using appropriate kits, following manufacturer's instructions. Serum NEFA were determined using a NEFA C kit (catalogue no. 994-75409E; Wako Chemicals, Richmond, VA, USA). Alanine aminotransferase (ALT) activity was determined using an ALT activity assay kit (catalogue no. K752-100; BioVision, Milpitas, CA, USA) to determine liver function and health. Glucose and insulin concentrations were used to calculate the HOMA of insulin resistance (HOMA-IR), a reliable marker of insulin sensitivity [21], which is defined as: fasting glucose $(\mathrm{mmol} / \mathrm{l}) \times$ fasting insulin $(\mathrm{mU} / \mathrm{l}) / 22.5$. Assays were measured with a Spectramax Plus 384 spectrophotometer (Molecular Devices, Sunnyvale, CA, USA).

Liver triacylglycerols Pieces of liver measuring 100 mg were cut and weighed using analytical scales. PBS $1 \mathrm{ml}$ was added to each tube and homogenised. Samples were centrifuged for $15 \mathrm{~s}$ at room temperature. The top layer was resuspended with gentle shaking. The supernatant fraction was transferred (without disturbing the pellet) to new $1.5 \mathrm{ml}$ tubes. Triacylglycerols were then assayed using a kit, following manufacturer's instructions (catalogue no. 17628; Sentinel Diagnostics).

Glycogen determination Two $\sim 20 \mathrm{mg}$ pieces of liver were cut and placed into $2 \mathrm{ml}$ tubes; $500 \mu \mathrm{l} 2 \mathrm{~mol} / \mathrm{l} \mathrm{HCl}$ was added to half of the samples and $500 \mu 12 \mathrm{~mol} / \mathrm{l} \mathrm{NaOH}$ (to control for free glucose) was added to the other half. All samples were heated for $2 \mathrm{~h}$ at $95^{\circ} \mathrm{C}$, following which $500 \mu \mathrm{mol} / \mathrm{l}$ $\mathrm{NaOH}$ was added to each tube containing $\mathrm{HCl}$ and $500 \mu \mathrm{l}$ $2 \mathrm{~mol} / \mathrm{l} \mathrm{HCl}$ was added to each tube containing $\mathrm{NaOH}$ for neutralisation. Tubes were vortexed and centrifuged for $1 \mathrm{~min}$. Samples were diluted 1:50 in equal volumes of $\mathrm{HCl}$ and $\mathrm{NaOH} ; 10 \mu \mathrm{l}$ of diluted samples was used for the assay. Hexokinase reagent $150 \mu$ l (Sigma) was added to each well and incubated for $10 \mathrm{~min}$ at room temperature. The assay was measured at $340 \mathrm{~nm}$ with a Spectramax Plus $384 \mathrm{spec}-$ trophotometer (Molecular Devices).

Glucose and pyruvate tolerance tests For glucose tolerance tests (GTTs), mice were injected i.p., following a $16 \mathrm{~h}$ fast, with glucose $1.5-2 \mathrm{mg} / \mathrm{g}$ body weight (1.5 $\mathrm{mg}$ for HFD-fed mice and $2 \mathrm{mg}$ for mice on chow diet). For pyruvate tolerance tests (PTTs), mice were injected with $1.5 \mathrm{mg} / \mathrm{g}$ pyruvate. Tail blood glucose values were measured using glucometers (AlphaTRAK, Abbott Laboratories, Abbott 
Park, IL, USA) immediately before and at 15, 30, 60 and 90 or $120 \mathrm{~min}$ after injection.

Immunoblotting Tissue lysates were prepared in radioimmunoprecipitation assay buffer containing fresh sodium orthovanadate and protease inhibitors, as described previously [22]. Proteins were separated by $4-12 \%$ SDS-PAGE and transferred to nitrocellulose membranes. Immunoblots were performed using antibodies from Cell Signaling Technology (New England Biolabs, Hitchin, UK) (unless stated otherwise) against PTP1B (Millipore), $\beta$-actin (Thermo Scientific), SH2 domain-containing protein tyrosine phosphatase 2 (SHP2; Santa Cruz, Dallas, TX, USA), phosphorylated glycogen synthase (p-GS) S641, phosphorylated glycogen synthase kinase 3 (p-GSK-3) $\alpha / \beta$ S21/S9, p-IR Y1162/63 (Invitrogen), p-IR Y1158 (Invitrogen, Carlsbad, CA, USA), p-Akt/protein kinase B (PKB) S473, phosphorylated extracellular signalregulated kinase ( $\mathrm{p}$-ERK)1/2 mitogen-activated protein kinase (MAPK) T202/Y204, p-Akt/PKB T308, p-FKBP-12rapamycin associated protein (FRAP)/phosphorylated mammalian target of rapamycin (p-mTOR) S2448 (Santa Cruz), p-S6 ribosomal protein S235/236, phosphorylated eukaryotic translation initiation factor- $2 \alpha$ kinase 3 (p-PERK) T980, phosphorylated eukaryotic initiation factor $2 \alpha$ (p-eIF2 $\alpha$ ) S51, phosphorylated stress-activated protein kinase (p-SAPK)/c-Jun $\mathrm{NH}_{2}$-terminal kinase (JNK) T183/Y185, ERK2 and total Akt/PKB (Santa Cruz). Immunoblots were developed using horseradish peroxidase-conjugated secondary antibodies, visualised using enhanced chemiluminescence, and quantified by densitometry scanning with Image $\mathrm{J}$ or BiolD software (PeqLab, Fareham, UK).

Gene expression analysis Total RNA was isolated from mouse liver and epididymal adipose tissue using TRI Reagent (Ambion, Warrington, UK), according to the manufacturer's protocol. First-strand cDNA was synthesised from $1 \mu \mathrm{g}$ total RNA employing the Bioline Bioscript Pre-amplification System (Bioline, London, UK) and oligo(dT) $)_{12-18}$. Target genes were amplified by real-time RT-PCR using $2 \mu$ diluted cDNA $(1: 10 ; 10 \mu \mathrm{l})$, using GoTaq qPCR Master Mix (Promega, Southampton, UK). The Roche LightCycler 480 System (Roche Diagnostics, Burgess Hill, UK) was used for analysis. Relative gene expression was calculated using the comparative $\mathrm{Ct}\left(2^{-\Delta \Delta C_{t}}\right)$ method. A geometric mean of three commonly used reference genes, Hprt (encoding hypoxanthine-guanine phosphoribosyltransferase), $18 S$ (encoding $18 \mathrm{~S}$ ribosomal RNA) and Gapdh (encoding glyceraldehyde 3-phosphate dehydrogenase), was used to normalise data. Geometric means of the relative copy numbers of mouse PCRs were followed by melting curves $\left(70-95^{\circ} \mathrm{C}\right)$.

Data analysis Data are expressed as mean $\pm \mathrm{SEM}$, and $n$ represents the number of mice or biological replicates.
Statistical analyses were performed using one-way ANOVA with Tukey's multiple comparison post hoc tests, repeated measures two-way ANOVA with Bonferroni multiple comparison post hoc tests, one-tailed $t$ tests or two-tailed Student's $t$ tests, as appropriate. The critical alpha level $(p)$ was set at $0.05 ; p<0.05$ was considered statistically significant. GraphPad Prism 5 statistical software was used for analyses (www.graphpad.com/scientific-software/prism/).

\section{Results}

Inducible liver-specific PTP1B knockdown improves glucose homeostasis Body weight (Fig. 1) was comparable between HFD-fed $S A-P t p 1 b^{-/}$and HFD-fed $P t p 1 b^{\mathrm{fl} / \mathrm{fl}}$ control mice throughout the study, and a decrease in PTP1B levels of $\sim 50 \%$ was achieved 4 and 12 weeks after tamoxifen treatment in HFD-fed SA-Ptp $1 b^{-1-}$ mice (Fig. 1b, c, respectively). PTP1B activity was also $20 \%$ lower in HFD-fed $S A-P t p 1 b^{-1-}$ mice compared with HFD-fed $P t p 1 b^{\mathrm{fl} / \mathrm{fl}}$ control mice (Fig. 1d). Considering that PTP1B activity was $\sim 40 \%$ lower in $A l b-P t p 1 b^{-1-}$ mice (which have Ptp $1 b$ deletion in the liver from birth) (Fig. 1d), this would be consistent with
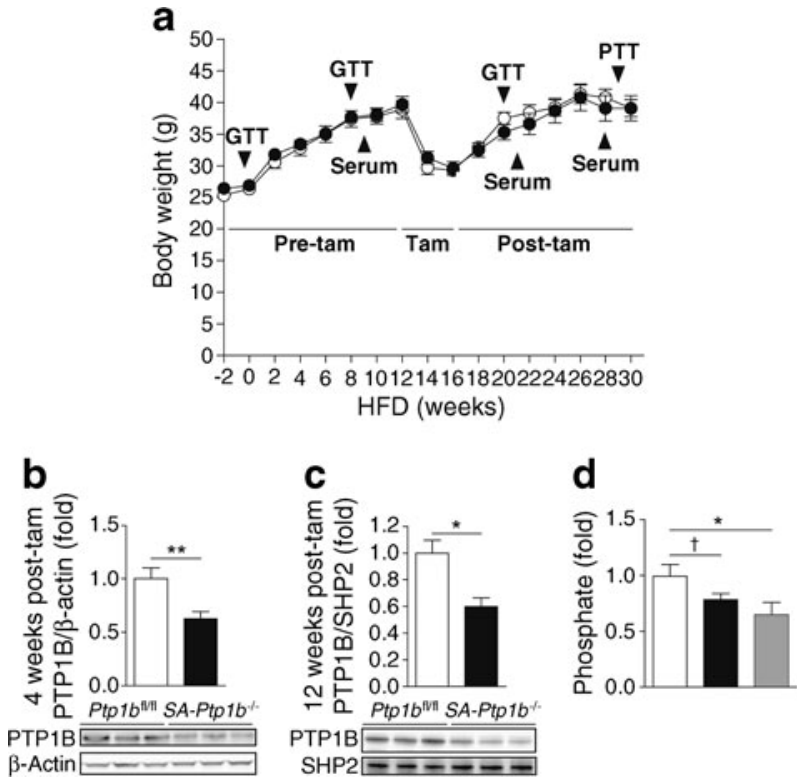

Fig. 1 Body weight and PTP1B knockdown/activity. (a) Body weight of HFD-fed $S A-P t p 1 b^{-/}(n=8)$ and HFD-fed Ptp1b $b^{\mathrm{fl} / \mathrm{fl}}$ control mice $(n=9)$. Experimental design and timings of tamoxifen (tam) treatment are also displayed. (b) PTP1B knockdown 4 weeks post-tamoxifen treatment $(n=4-7)$. (c) PTP1B knockdown 12 weeks post-tamoxifen treatment $(n=4-7)$. (d) PTP1B activity 12 weeks post-tamoxifen treatment. HFD-fed $P t p 1 b^{\mathrm{fl} / \mathrm{fl}}$ control mice $(n=8)$, HFD-fed $S A$ $P t p 1 b^{-1-}$ mice $(n=8)$ and HFD-fed Alb-Ptplb ${ }^{-1-}$ mice $(n=4)$. Data are represented as mean \pm SEM. White bars/white circles, $P t p 1 b^{\mathrm{fl} / \mathrm{fl} \text {; }}$ black bars/black circles, $S A-P t p 1 b^{-1-}$; grey bar, Alb-Ptp $1 b^{-/-}$. Data were analysed by one-tailed or two-tailed Student's $t$ test $\left({ }^{*} p<0.05\right.$; $* * p<0.01 ;{ }^{\dagger} p=0.054$ ) 
$50 \%$ deletion at the protein level. As expected from other studies, tamoxifen treatment caused $\sim 20 \%$ body weight loss in both groups of mice, which returned to pre-tamoxifen levels on HFD (Fig. 1). An outline of the experimental design is shown in Fig. 1. As expected, HFD-fed $S A$ $P t p 1 b^{-/-}$mice displayed no differences in glucose tolerance in comparison with $P t p 1 b^{\mathrm{fl} / \mathrm{fl}}$ mice prior to tamoxifen treatment, on a chow or HFD diet (Fig. 2a, b). Importantly, HFD feeding induced glucose intolerance in both groups of mice, as evidenced by an increased AUC in both groups (Fig. 2d). After tamoxifen treatment, which induced PTP1B knockdown in $S A-P t p 1 b^{-/}$mice only (Fig. 1b, c), HFD-fed $S A$ $P t p 1 b^{-/-}$mice displayed a significantly improved response to glucose challenge and a reversal of glucose intolerance to chow diet feeding levels (Fig. 2a, c, d). Furthermore, fed and fasting serum glucose levels were lower in HFD-fed $S A$ $P t p 1 b^{-/-}$mice compared with HFD-fed $P t p 1 b^{\mathrm{fl} / \mathrm{fl}}$ control mice 4 and 12 weeks after tamoxifen treatment (Fig. 2f, g). Fed serum insulin levels tended to be lower in $S A-P t p 1 b^{-1-}$ mice, although this did not reach significance (Fig. 2h). Fasting serum insulin levels, however, were significantly lower 4 weeks after tamoxifen treatment in HFD-fed $S A-P t p 1 b^{-1-}$ mice compared with HFD-fed Ptp $1 b^{\mathrm{f} / \mathrm{fl}}$ controls (Fig. 2i). Importantly, HOMA-IR, which represents an index of insulin resistance, was significantly lower in HFD-fed SA-Ptplb $b^{-/-}$mice 4 and 12 weeks after tamoxifen treatment (Fig. 2j).

Inducible liver-specific PTP1B knockdown improves lipid homeostasis Haematoxylin and eosin staining revealed a high level of inter-animal variability when examining lipid deposition in livers of HFD-fed $S A-P t p 1 b^{-1}$ and $P t p 1 b^{\mathrm{fl} / \mathrm{fl}}$ mice (Fig. 3a). We therefore also assessed total liver triacylglycerol levels, which revealed that they were significantly lower in the livers of HFD-fed $S A-P t p 1 b^{-1-}$ mice compared with HFD-fed $P t p 1 b^{\mathrm{fl} / \mathrm{fl}}$ controls (Fig. 3b). There were no significant differences between groups in ALT activity (Fig. 3c). Fed serum NEFA were significantly decreased 4 and 12 weeks after tamoxifen treatment in HFDfed $S A-P t p 1 b^{-/}$mice compared with $P t p 1 b^{\mathrm{fl} / \mathrm{fl}}$ controls (Fig. 3d). HFD-fed $S A-P t p 1 b^{--}$mice also displayed
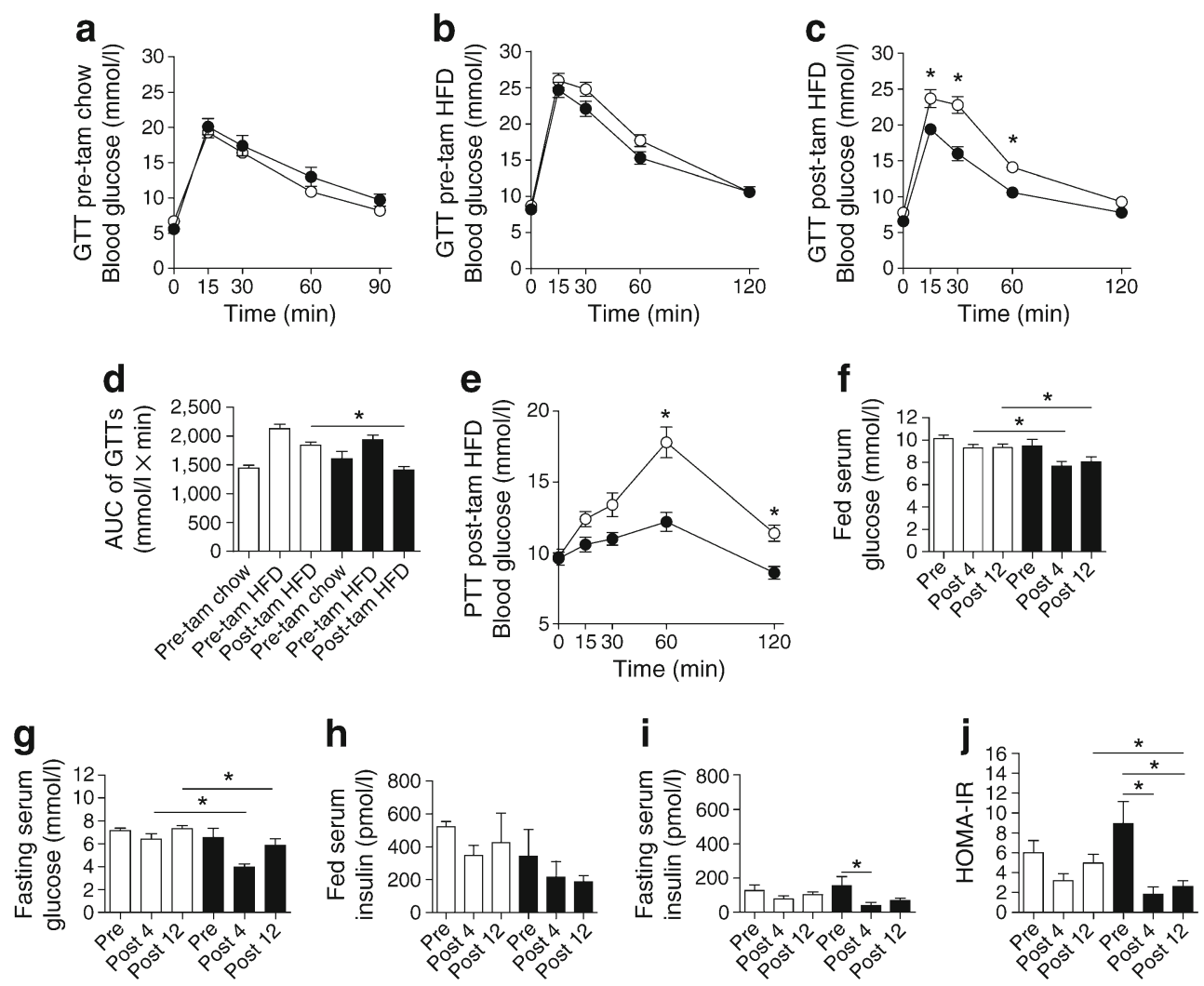

Fig. 2 Inducible liver-specific PTP1B knockdown improves glucose homeostasis. (a) GTT of both groups on chow diet prior to tamoxifen (tam) treatment. (b) GTT of both groups on HFD for 8 weeks prior to tamoxifen treatment. (c) GTT of both groups on HFD 3 weeks after tamoxifen treatment. (d) AUC of GTTs. (e) PTT of both groups on HFD 12 weeks after tamoxifen treatment. (f) Fed serum glucose levels. (g) Fasting serum glucose levels. (h) Fed serum insulin levels. (i) Fasting serum insulin levels. (j) HOMA-IR. SA-Ptp $1 b^{-1-}$ and Ptp $1 b^{\mathrm{fl} / \mathrm{fl}}$ control

groups are indicated in the figures. For all experiments $n=8$ for HFDfed $S A-P t p 1 b^{-/-}$mice and $n=9$ for HFD-fed $P t p 1 b^{\mathrm{fl} / \mathrm{fl}}$ control mice. Data are represented as mean \pm SEM. White bars/white circles, $P t p 1 b^{\mathrm{fl} / \mathrm{fl}}$; black bars/black circles, $S A-P t p 1 b^{-/-}$. Data were analysed by repeated measures two-way ANOVA with Bonferroni multiple comparison post hoc tests, one-way ANOVA with Tukey's multiple comparison post hoc tests or two-tailed Student's $t$ test, where appropriate $\left({ }^{*} p<0.05\right)$ 
Fig. 3 Lipid homeostasis is improved with inducible liverspecific PTP1B knockdown. (a) Haematoxylin and eosin staining of livers. (b) Liver triacylglycerol assay from HFDfed $S A-P t p 1 b^{-/-}$mice and HFDfed $P \operatorname{tp} 1 b^{\mathrm{fl} / \mathrm{fl}}$ control mice. (c) ALT activity assay. (d) Fed serum NEFA assay. (e) Fasting serum NEFA assay. (f) Fed serum triacylglycerol assay. (g) Fasting serum triacylglycerol assay. (h) Fed circulating leptin assay. (i) Fasting circulating leptin assay. $S A-P t p 1 b^{-/-}$and $P \operatorname{tp} 1 b^{\mathrm{fl} / \mathrm{fl}}$ control groups are indicated in the figures. For all experiments $n=8$ for HFD-fed $S A-P t p 1 b^{-1}$ mice and $n=9$ for HFD-fed $P t p 1 b^{\mathrm{fl} / \mathrm{fl}}$ control mice. Data are represented as mean \pm SEM. White bars, $P t p 1 b^{\mathrm{fl} / \mathrm{fl}}$; black bars, SA-Ptp $1 b^{-1-}$. Data were analysed by one-way ANOVA with Tukey's multiple comparison post hoc tests or two-tailed Student's $t$ test, where appropriate $(* p<0.05)$ a
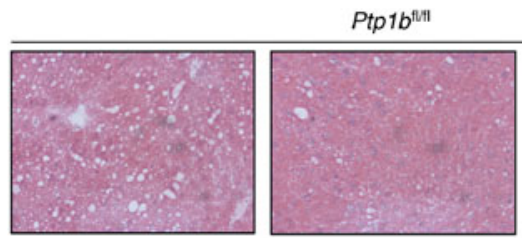

SA-Ptp $1 b^{*}$

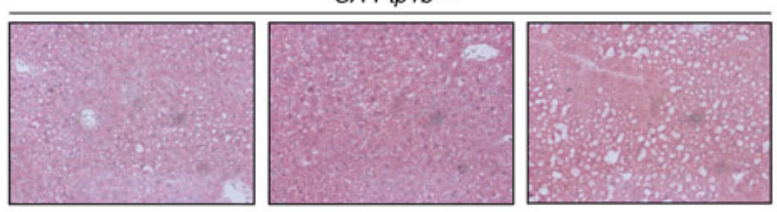

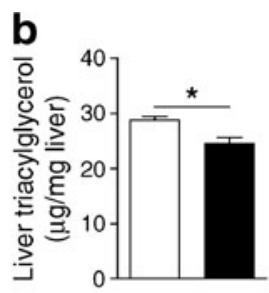
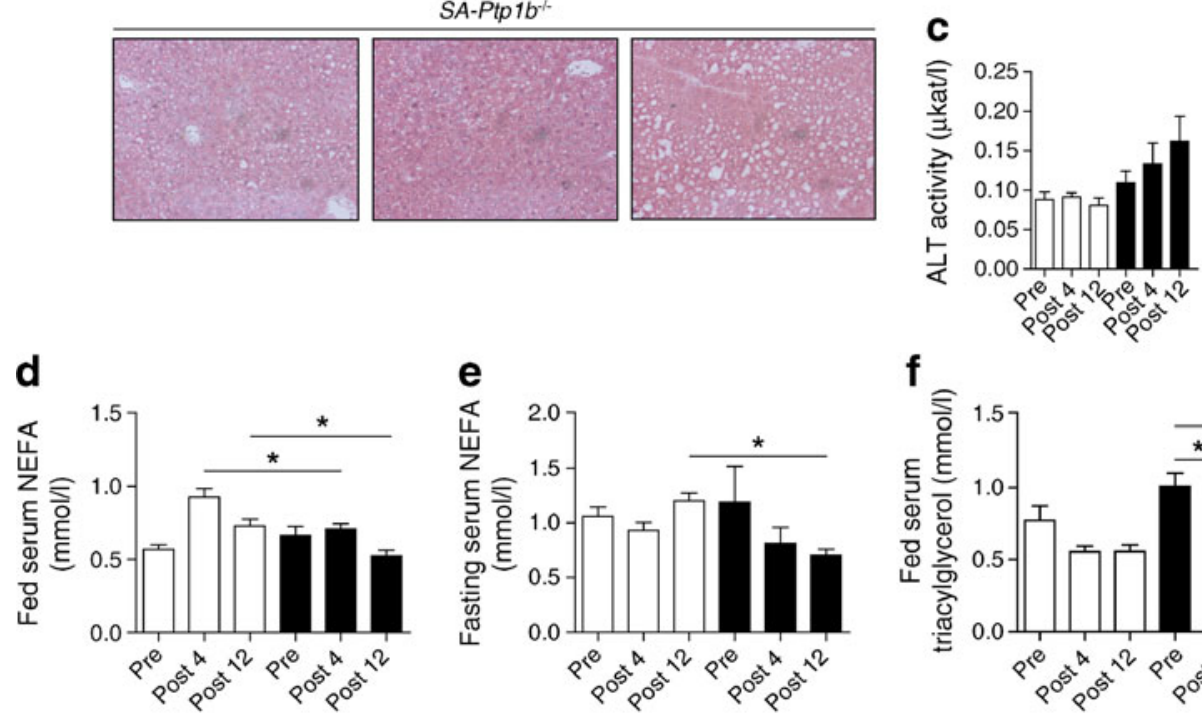

f

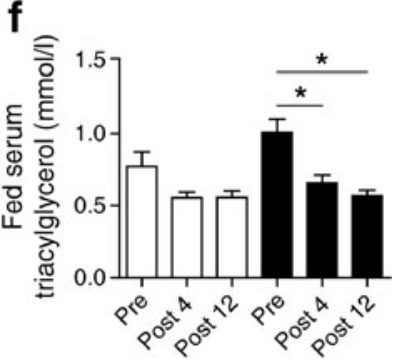

h

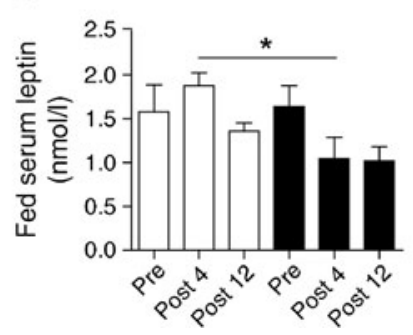

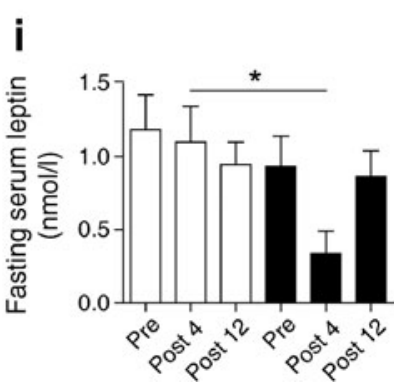

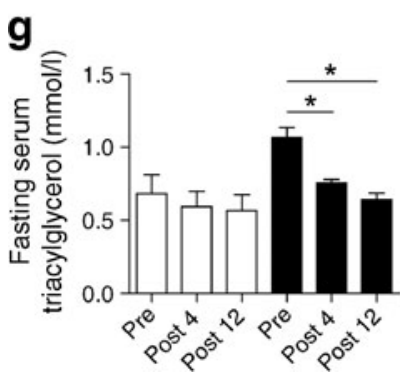

significantly lower fasting serum NEFA 12 weeks after tamoxifen treatment (Fig. 3e). Fed and fasting serum triacylglycerol levels were significantly decreased both 4 and 12 weeks after tamoxifen treatment compared with pre-tamoxifen levels in HFD-fed $S A-P t p 1 b^{-1-}$ mice only (Fig. 3f, g). Moreover, both fed and fasting serum leptin levels were significantly lower after 4 weeks of tamoxifen treatment in HFD-fed $S A-P t p 1 b^{-1}$ mice compared with HFD-fed $P t p 1 b^{\mathrm{fl} / \mathrm{fl}}$ control mice (Fig. 3h, i). Furthermore, IL-6, MCP-1, resistin and TNF- $\alpha$ were measured in $P t p 1 b^{\mathrm{fl} / \mathrm{fl}}$ control and $S A-P t p 1 b^{-/-}$mice in the fasted state (Table 1). SA-Ptp $1 b^{-/-}$mice displayed lower IL-6 after 12 weeks of tamoxifen treatment compared with $P t p 1 b^{\mathrm{fl} / \mathrm{fl}}$ control mice ( $p=0.059$; two-tailed $t$ test), whilst MCP-1 was increased in $S A$-Ptplb ${ }^{-/}$mice 4 weeks after tamoxifen treatment compared with $P t p 1 b^{\mathrm{fl} / \mathrm{fl}}$ control mice. Resistin was not different between groups at any time point (Table 1) and TNF- $\alpha$ concentrations were below the level of detection of the mouse multiplex ELISA (data not shown).
Table 1 Serum glucose levels of IL-6, MCP-1 and resistin before and after 4 and 12 weeks of tamoxifen treatment

\begin{tabular}{lll}
\hline Variable & $\operatorname{Ptplb}^{\mathrm{fl} / \mathrm{fl}}(n=6)$ & SA-Ptplb $^{-/-}(n=6)$ \\
\hline IL-6 (pmol/1) & & \\
$\quad$ Before treatment & $1.04 \pm 0.39$ & $0.65 \pm 0.18$ \\
After 4 weeks & $0.31 \pm 0.08$ & $0.71 \pm 0.32$ \\
After 12 weeks & $1.43 \pm 0.43$ & $0.5 \pm 0.07^{\dagger}$ \\
MCP-1 (pmol/1) & & \\
Before treatment & $1.53 \pm 0.1$ & $1.27 \pm 0.26$ \\
After 4 weeks & $0.64 \pm 0.26$ & $2.82 \pm 0.63^{*}$ \\
After 12 weeks & $1.17 \pm 0.47$ & $1.81 \pm 0.52$ \\
Resistin (pmol/1) & & \\
Before treatment & $108.13 \pm 21.47$ & $83.28 \pm 15.55$ \\
After 4 weeks & $102.38 \pm 14.18$ & $81.90 \pm 22.28$ \\
After 12 weeks & $77.04 \pm 17.38$ & $79.68 \pm 13.53$ \\
\hline
\end{tabular}

Data are represented as mean \pm SEM and were analysed using one-way ANOVA with Tukey's multiple comparison post hoc tests $\left({ }^{*} p<0.05\right.$; $\left.{ }^{\dagger} p=0.059\right)$ 
Inducible liver-specific PTP1B knockdown improves suppression of hepatic gluconeogenesis To assess if most of the improvements in glucose homeostasis were due to improvements in suppression of hepatic gluconeogenesis, we performed a PTT. HFD-fed $S A-P t p 1 b^{-/-}$mice displayed a significantly improved response to pyruvate challenge following overnight fasting compared with HFD-fed Ptp $1 b^{\mathrm{fl} / \mathrm{fl}}$ controls (Fig. 2e). This is consistent with increased insulininduced GS dephosphorylation after both 4 and 12 weeks of tamoxifen treatment (Fig. 4a-d). Liver glycogen content was unaltered between $S A-P t p 1 b^{-/}$and $P t p 1 b^{\mathrm{fl} / f 1}$ control mice (5.97 \pm 3.41 vs $8.32 \pm 2.93 \mu \mathrm{g} / \mathrm{mg}$, respectively). Surprisingly, components of the classical insulin signalling pathway were unchanged with liver PTP1B knockdown both 4 and 12 weeks after tamoxifen treatment (Fig. 4a, b, e). Furthermore, insulin signalling in muscle and epididymal white adipose tissue (WAT) was comparable between $S A-P t p 1 b^{-/-}$ and $P t p 1 b^{\mathrm{fl} / \mathrm{fl}}$ control mice (Fig. 4f, g).

Inducible liver-specific PTP1B knockdown is associated with decreased expression of liver gluconeogenic genes To assess the mechanism(s) behind improvements in whole-

a

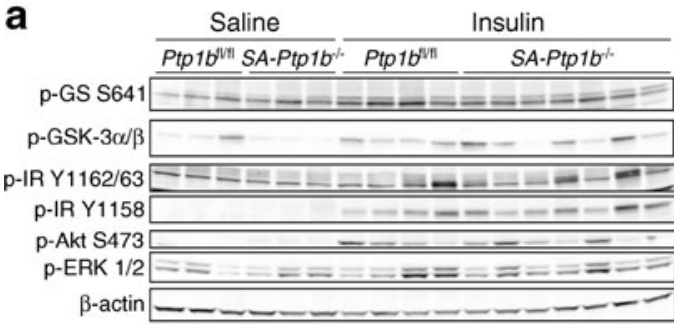

C
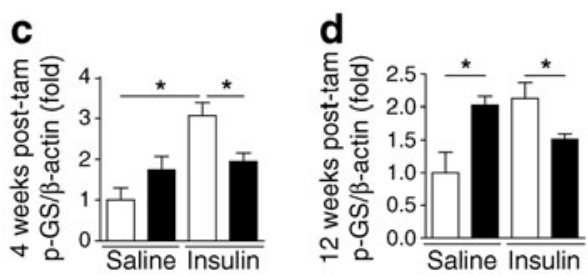

f

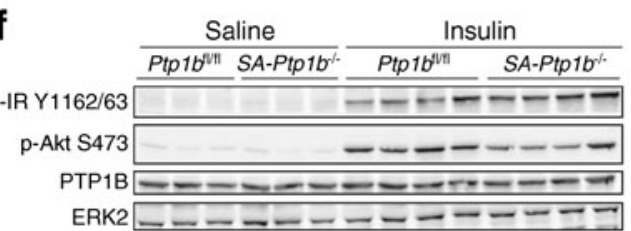

Fig. 4 Inducible liver-specific PTP1B knockdown improves suppression of hepatic gluconeogenesis. (a) Liver insulin signalling 4 weeks post-tamoxifen (tam) in HFD-fed Ptpl $1 b^{\mathrm{fl} / \mathrm{fl}}$ and HFD-fed $S A$-Ptp $1 b^{-/}$ mice after injection with saline or insulin $(10 \mathrm{mU} / \mathrm{g})$. (b) Liver insulin signalling 12 weeks post-tamoxifen in HFD-fed $P t p 1 b^{\mathrm{fl} / \mathrm{fl}}$ and HFD-fed $S A-P t p 1 b^{-/}$mice after injection with saline or insulin $(10 \mathrm{mU} / \mathrm{g})$. (c) Quantification of GS immunoblot 4 weeks post-tamoxifen: HFD-fed $P t p 1 b^{\mathrm{fl} / f \mathrm{l}}$ control mice, $n=7$ (three saline/four insulin); HFD-fed $S A$ $P t p 1 b^{-/-}$mice, $n=10$ (three saline/seven insulin). (d) Quantification of GS immunoblot 12 weeks post-tamoxifen: HFD-fed $P t p 1 b^{-\mathrm{fl} / \mathrm{fl}}$ control mice, $n=7$ (three saline/four insulin); HFD-fed $S A-P t p 1 b^{-/-}$mice, $n=10$ body glucose and lipid homeostasis, we analysed the expression of genes involved in gluconeogenesis and lipogenesis. Consistent with the physiological data from PTTs (Fig. 2e) and signalling data (Fig. 4a-d), HFD-fed $S A$ $P t p 1 b^{-1-}$ mice displayed a decrease in gluconeogenic markers in comparison with HFD-fed $P t p 1 b^{\mathrm{fl} / \mathrm{fl}}$ control mice, as evidenced by decreased liver gene expression levels of Pepck (Table 2). Moreover, Pparg was significantly decreased in these mice (Table 2). Furthermore, Hmgcs 1 was increased in HFD-fed $S A-P t p 1 b^{-/-}$mice compared with HFD-fed $P t p 1 b^{\mathrm{fl} / \mathrm{fl}}$ control mice (Table 1). HFD-fed $S A$ $P t p 1 b^{-/-}$mice exhibited unaltered lipogenic gene expression in liver or epididymal WAT compared with $P t p 1 b^{\mathrm{fl} / \mathrm{fl}}$ control mice (Table 1). Lipolytic and adipokine gene expression levels were unaltered between the groups in epididymal WAT (Table 2).

Inducible liver-specific PTP1B knockdown decreases ER stress At the gene expression level, there was a significant decrease in Grp94 in HFD-fed SA-Ptp $1 b^{-1-}$ mice compared with HFD-fed $P t p 1 b^{\mathrm{fl} / \mathrm{fl}}$ control mice (Table 2). At the protein level, HFD-fed $S A-P t p 1 b^{-/-}$mice exhibited significantly
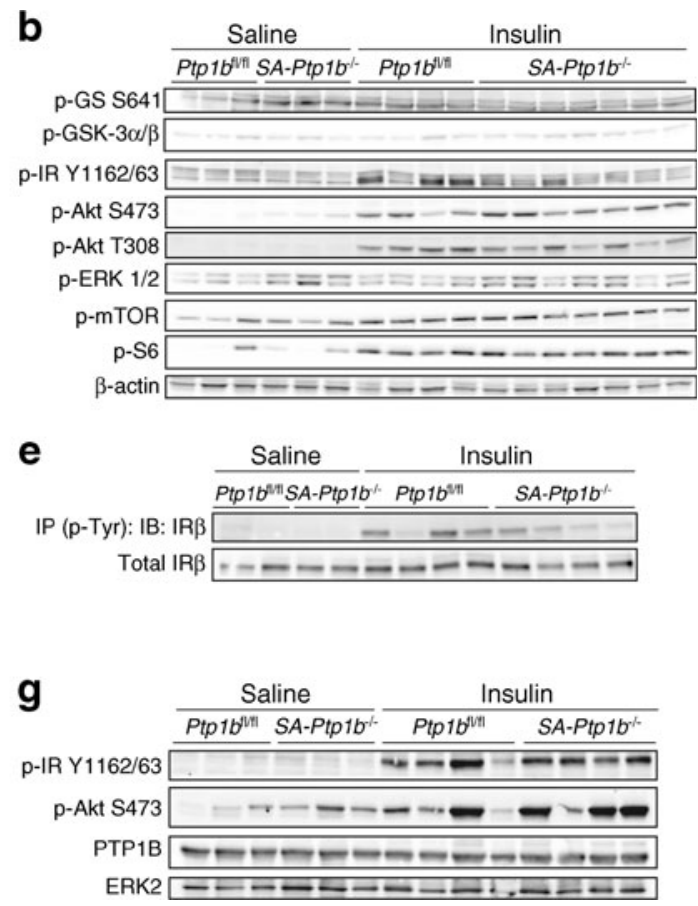

(three saline/seven insulin). (e) Liver IR phosphorylation by immunoprecipitation 12 weeks post-tamoxifen: HFD-fed $P \operatorname{tp} 1 b^{\mathrm{fl} / \mathrm{fl}}$ control mice, $n=6$ (two saline/four insulin); HFD-fed $S A-P t p 1 b^{-/-}$mice, $n=6$ (two saline/four insulin). (f) Muscle insulin signalling 12 weeks post-tamoxifen. (g) Epididymal WAT insulin signalling 12 weeks post-tamoxifen: HFD-fed $P t p 1 b^{\mathrm{fl} / \mathrm{fl}}$ control mice, $n=7$ (three saline/four insulin); HFDfed $S A-P t p 1 b^{-/-}$mice, $n=7$ (three saline/four insulin). Data are represented as mean \pm SEM. White bars, $P t p 1 b^{\mathrm{fl} / \mathrm{fl}}$; black bars, $S A-P t p 1 b^{-/-}$. Data were analysed by one-way ANOVA with Tukey's multiple comparison post hoc tests or two-tailed Student's $t$ test, where appropriate $\left({ }^{*} p<0.05\right)$ 
Table 2 Lipid and glucose metabolism gene expression in liver and epididymal WAT

\begin{tabular}{|c|c|c|c|}
\hline Variable & $\begin{array}{l}P t p 1 b^{\mathrm{fl} / \mathrm{fl}} \\
(n=6-7)\end{array}$ & $\begin{array}{l}\text { SA-Ptp } 1 b^{-/-} \\
(n=6-8)\end{array}$ & $p$ value \\
\hline \multicolumn{4}{|c|}{ Liver metabolism } \\
\hline Fas & $1.0 \pm 0.06$ & $1.0 \pm 0.23$ & 0.983 \\
\hline Srebplc & $1.0 \pm 0.11$ & $1.4 \pm 0.24$ & 0.155 \\
\hline Srebpla & $1.0 \pm 0.22$ & $1.3 \pm 0.22$ & 0.380 \\
\hline Srebp2 & $1.0 \pm 0.20$ & $1.1 \pm 0.23$ & 0.751 \\
\hline Hmgcs1 & $1.0 \pm 0.14$ & $1.9 \pm 0.32 *$ & 0.023 \\
\hline Pparg & $1.0 \pm 0.12$ & $0.6 \pm 0.08^{*}$ & 0.023 \\
\hline Ppara & $1.0 \pm 0.14$ & $0.8 \pm 0.12$ & 0.307 \\
\hline Pgcla & $1.0 \pm 0.13$ & $0.7 \pm 0.07$ & 0.056 \\
\hline Pepck & $1.0 \pm 0.14$ & $0.6 \pm 0.05^{*}$ & 0.018 \\
\hline$G 6 p$ & $1.0 \pm 0.26$ & $0.7 \pm 0.15$ & 0.289 \\
\hline \multicolumn{4}{|l|}{ Liver ER stress } \\
\hline Bip & $1.0 \pm 0.08$ & $0.9 \pm 0.08$ & 0.317 \\
\hline Grp94 & $1.0 \pm 0.12$ & $0.6 \pm 0.10^{*}$ & 0.043 \\
\hline Chop & $1.0 \pm 0.05$ & $1.1 \pm 0.22$ & 0.667 \\
\hline Xbp Spliced & $1.0 \pm 0.13$ & $1.3 \pm 0.16$ & 0.241 \\
\hline Xbp Total & $1.0 \pm 0.08$ & $1.2 \pm 0.15$ & 0.268 \\
\hline Atf4 & $1.0 \pm 0.06$ & $1.1 \pm 0.09$ & 0.296 \\
\hline \multicolumn{4}{|c|}{ Adipose tissue metabolism } \\
\hline Fas & $1.0 \pm 0.11$ & $0.8 \pm 0.14$ & 0.303 \\
\hline Srebplc & $1.0 \pm 0.20$ & $1.2 \pm 0.21$ & 0.605 \\
\hline Srebpla & $1.0 \pm 0.15$ & $1.1 \pm 0.13$ & 0.565 \\
\hline Pparg & $1.0 \pm 0.10$ & $0.9 \pm 0.16$ & 0.622 \\
\hline Ppara & $1.0 \pm 0.27$ & $1.2 \pm 0.43$ & 0.775 \\
\hline$H s l$ & $1.0 \pm 0.17$ & $0.9 \pm 0.13$ & 0.673 \\
\hline Atgl & $1.0 \pm 0.17$ & $0.7 \pm 0.11$ & 0.150 \\
\hline Rbp4 & $1.0 \pm 0.15$ & $0.8 \pm 0.19$ & 0.461 \\
\hline Leptin & $1.0 \pm 0.27$ & $0.9 \pm 0.29$ & 0.820 \\
\hline Adipoq & $1.0 \pm 0.15$ & $0.6 \pm 0.20$ & 0.185 \\
\hline Resistin & $1.0 \pm 0.23$ & $0.9 \pm 0.23$ & 0.792 \\
\hline Glut4 & $1.0 \pm 0.17$ & $0.8 \pm 0.12$ & 0.315 \\
\hline Pepck & $1.0 \pm 0.17$ & $0.7 \pm 0.19$ & 0.272 \\
\hline$F 480$ & $1.0 \pm 0.44$ & $0.7 \pm 0.38$ & 0.652 \\
\hline
\end{tabular}

Data are represented as mean \pm SEM and presented as fold change relative to $P \operatorname{tp} 1 b^{\mathrm{fl} / \mathrm{fl}}$ group; data were analysed using two-tailed Student's $t$ test $(* p<0.05)$

lower phosphorylation of PERK, eIF2 $\alpha$ and JNK2 when compared with $P t p 1 b^{\mathrm{fl} / \mathrm{fl}}$ control mice (Fig. 5a-d).

\section{Discussion}

There is a growing body of evidence to suggest that PTP1B inhibitors hold great promise for the treatment of type 2 diabetes as well as cancer [3, 4, 8, 16-18, 22-30]. Numerous
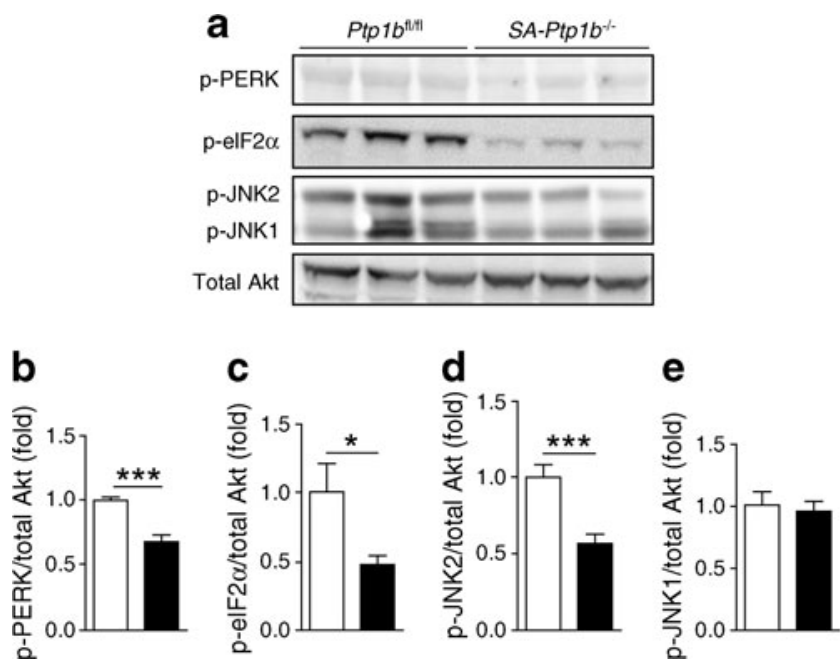

Fig. 5 Inducible liver-specific PTP1B knockdown reduces ER stress. (a) Representative blot of liver ER stress signalling 12 weeks post-tamoxifen treatment in HFD-fed $P t p 1 b^{\mathrm{fl} / \mathrm{fl}}$ and HFD-fed $S A-P t p 1 b^{-/-}$mice. (b-e) Quantification of p-PERK, p-eIF2 $\alpha$, p-JNK2 and p-JNK1 immunoblots: HFD-fed $P t p 1 b^{\mathrm{fl} / \mathrm{fl}}$ control mice, $n=7$; HFD-fed $S A$-Ptp $1 b^{-/-}$mice, $n=10$. Data are represented as mean $\pm \mathrm{SEM}$. White bars, $P t p 1 b^{\mathrm{fl} / \mathrm{fl}}$; black bars, $S A-P t p 1 b^{-/ \tau}$. Data were analysed by two-tailed Student's $t$ test $\left({ }^{*} p<0.05\right.$; $* * * p<0.001)$

mouse and human studies have demonstrated that decreasing PTP1B in various tissues including muscle, liver and brain leads to a multitude of beneficial effects $[17,18,22,31]$. Liver-specific $P t p 1 b$ knockout in mice $\left(A l b-P t p 1 b^{-1-}\right)$ led to improved glucose homeostasis and decreased triacylglycerol levels independently of changes in body weight [17]. Previous studies, however, investigated mice with a knockout of Ptp $1 b$ from birth and have therefore examined the effects of Ptplb deletion in preventing type 2 diabetes but not in treating already obese and insulin-resistant states. Using a tamoxifen-dependent Cre recombinase system, we now demonstrate that decreasing liver PTP1B by $~ 50 \%$ in obese and insulin-resistant adult mice leads to a reversal of glucose intolerance and improvements in lipid homeostasis, and that these effects are manifested within just a matter of weeks after hepatic PTP1B knockdown.

As expected, and reported by others, oral tamoxifen treatment caused a transient decrease in body weight in both groups of mice [32-34]. As with other mouse models of Ptp1b-specific deletion [17, 22], body weight of the inducible liver-specific Ptp $1 b$ knockout mice did not differ from that of control mice. PTP1B knockdown decreased PTP1B protein levels by $\sim 50 \%$ and PTP1B activity by $\sim 20 \%$ in livers from $S A-P t p 1 b^{-/}$mice. A $50 \%$ knockdown is less than was observed previously in livers from Alb-Ptp $1 b^{-/-}$ mice (achieving $~ 80 \%$ hepatic Ptp $1 b$ deletion and $\sim 40 \%$ activity inhibition) $[16,17]$. It has recently been reported that different Cre lines display different degrees of efficiency and specificity [35]. In addition to differences amongst Cre 
mice, different floxed gene loci were shown to display a range of sensitivity to recombination when using different Cre lines [35]. However, a 50\% decrease in PTP1B levels is physiologically relevant, as PTP1B inhibitors would only be expected to achieve approximately these levels [24].

In agreement with previous studies, glucose homeostasis is improved in SA-Ptp $1 b^{-/-}$mice compared with control mice [17]. Interestingly, glucose tolerance of $S A-P t p 1 b^{-/-}$ mice returned to the responsiveness measured in these mice on a chow diet, suggesting a reversal in glucose intolerance that was caused by 12 weeks of HFD feeding prior to inhibition of hepatic PTP1B. Furthermore, $S A-P t p 1 b^{-/-}$mice exhibited significantly lower blood glucose levels in response to a pyruvate bolus, suggesting an increased ability of insulin to suppress hepatic gluconeogenesis. Consistent with these physiological data and our previous studies using Alb-Ptp $1 b^{-1-}$ mice, we observed increased insulin-induced GS dephosphorylation and decreased expression of the gluconeogenic gene Pepck in livers of $S A-P t p 1 b^{-1-}$ mice, in the absence of changes in liver glycogen content [17]. This suggests that $S A-P t p 1 b^{-/-}$mice have an improved gluconeogenic response, efficiently shutting down hepatic glucose production compared with control mice.

It is interesting to note that in our experiment insulin treatment of the control mice led to increased GS phosphorylation, whilst in the $S A-P t p 1 b^{-/-}$mice it led to the expected dephosphorylation. At the moment, it is unclear how hepatic PTP1B inhibition affects GS phosphorylation independently of its effects on the IR; however, PTP1B has been shown to regulate protein phosphatase 2 ( $\mathrm{PP} 2 \mathrm{~A})$ activation [36] as well as regulate hepatic Srebp1 gene expression through the PP2A axis [37], which may then affect the GS hepatic phosphorylation state [38]. This is currently under investigation in our laboratory, but is consistent with data from $P t p 1 b^{-/}$immortalised cells treated with insulin, which were also shown to exhibit enhanced dephosphorylation of the S641 site on GS [39].

Liver Ptplb deletion has previously been shown to decrease serum triacylglycerol with lower expression of lipogenic genes [17]. This suggests that PTP1B knockdown may be a suitable therapy for NAFLD, which is characterised by increased hepatic lipid accumulation and insulin resistance. A recent study showed that the dietary supplement curcumin inhibits PTP1B and prevents hepatic steatosis in fructose-fed rats, providing support for this notion [40]. Here, we demonstrate that PTP1B knockdown in obese and diabetic mice results in lower liver triacylglycerol levels associated with decreased expression of Pparg, which has been found to be elevated in fatty livers [41].

Interestingly, a paradoxical phenotype was previously observed in Alb-Ptp $1 b^{-1-}$ mice, which displayed increased hepatic insulin signalling and decreased expression levels of hepatic Srebp1c, Fas and other lipogenic markers [16, 17]. It is suggested that PTP1B may affect Srebpl expression via a non-insulin-signalling pathway in the liver involving effects on PP2A activity $[23,37]$. No differences were noted in Srebp1a, Srebplc or Fas in the current study; it may be that a $50 \%$ PTP1B knockdown is not sufficient to measure detectable changes or it may be due to the differences in timing of the Ptplb deletion.

We have previously shown that $A l b-P t p 1 b^{-/-}$mice, which delete hepatic Ptp $1 b$ from birth, are protected against HFDinduced hepatic ER stress [17]. Consistent with this, $S A$ $P t p 1 b^{-/-}$mice also have decreased phosphorylation levels of PERK, eIF $2 \alpha$ and JNK2, indicating that $\sim 50 \%$ knockdown of PTP1B can temporally improve ER stress. Moreover, in the absence of changes in the classical IR signalling, this study suggests that improvements in lipid homeostasis observed with hepatic PTP1B knockdown may be due to decreased ER stress response signalling.

$S A-P t p 1 b^{-1-}$ mice displayed significantly lower fed and fasting leptin levels 4 weeks after PTP1B knockdown was induced. This is the first time that PTP1B knockdown in the liver has been reported to affect circulating leptin levels. PTP1B has been well documented to regulate leptin receptor signalling [18, 42]. Leptin action in the liver remains, however, inconclusive. Diet-induced obese rats have been shown to exhibit decreased hepatic levels of leptin receptor transcripts [43, 44]. Moreover, leptin treatment of wild-type mice led to increased mRNA expression of several isoforms of the leptin receptor, including the long form of the receptor $(\mathrm{Ob}-\mathrm{Rb})$ [45], suggesting that the liver may be an important site of leptin action. Furthermore, overexpression of PTP1B in the liver was shown to restrict the ability of leptin to lower blood glucose levels and suppress food intake [46]. It was suggested that strategies aimed at suppressing PTP1B specifically in the liver could improve both hepatic insulin and leptin sensitivity [46]. Revealing the mechanism(s) behind our current observations should form part of future studies.

Overall, tissue-specific knockout/knockdown studies of PTP1B have revealed key roles for brain, liver and muscle PTP1B in the regulation of global energy and glucose homeostasis. We now demonstrate that liver PTP1B knockdown not only prevents but can also reverse established insulin resistance and glucose intolerance, as well as decrease ER stress and fat accumulation in the liver in obese and insulin-resistant states. Inhibition of PTP1B remains a promising potential therapy for type 2 diabetes treatment as well as a potential protection against the development of NAFLD.

Acknowledgements We would like to thank P. Chambon and D. Metzger (Institut de Génétique et de Biologie Moléculaire et Cellulaire [IGBMC], C.U. de Strasbourg, Illkirch, France) for kindly providing $S A-C r e-E R^{T 2}$ mice. We would like to thank B. Neel (Campbell Family Cancer Research Institute, Ontario Cancer Institute, University of Toronto, ON, Canada) and B. Kahn (Division of Endocrinology, Diabetes 
and Metabolism, Beth Israel Deaconess Medical Centre, Boston, MA, USA) for Ptp $1 b$ floxed mice. We would also like to thank G. Mcilroy (Institute of Medical Sciences, School of Medical Sciences, University of Aberdeen, Aberdeen, UK) for helping out with GTTs.

Funding This work was supported by a British Heart Foundation project grant (PG/09/048/27675 and PG/11/8/28703) and a Diabetes UK project grant (BDA/RD08/0003597) awarded to M. Delibegović. C. Owen and E. K. Lees are recipients of BBSRC postgraduate studentships. N. Mody is the recipient of a British Heart Foundation intermediate basic research fellowship.

Duality of interest The authors declare that there is no duality of interest associated with this manuscript

Contribution statement $\mathrm{CO}, \mathrm{EKL}, \mathrm{LG}, \mathrm{NM}$ and MD contributed to the acquisition of data and performed the analyses. $\mathrm{CO}$ and EKL wrote the first draft of the paper, and LG, DJZ, NM, KKB and MD contributed to the interpretation of data and critical revision of the manuscript. All authors were involved in the writing of the manuscript and approved the final version of the article.

\section{References}

1. Simons P, van den Pangaart P, van Roomen C, Aerts J, Boon L (2005) Cytokine-mediated modulation of leptin and adiponectin secretion during in vitro adipogenesis: evidence that tumor necrosis factor-[alpha]-and interleukin-1 [beta]-treated human preadipocytes are potent leptin producers. Cytokine 32:94-103

2. Biddinger S, Kahn C (2006) From mice to men: insights into the insulin resistance syndromes. Ann Rev Physiol 68:123-158

3. Rondinone CM, Trevillyan JM, Clampit J et al (2002) Protein tyrosine phosphatase $1 \mathrm{~B}$ reduction regulates adiposity and expression of genes involved in lipogenesis. Diabetes 51:2405-2411

4. Zinker BA, Rondinone CM, Trevillyan JM et al (2002) PTP1B antisense oligonucleotide lowers PTP1B protein, normalizes blood glucose, and improves insulin sensitivity in diabetic mice. Proc Natl Acad Sci U S A 99:11357-11362

5. Kahn B (1995) Lilly lecture 1995. Glucose transport: pivotal step in insulin action. Diabetes 45:1644-1654

6. Shulman GI (2000) Cellular mechanisms of insulin resistance. J Clin Invest 106:171-176

7. Abel ED, Peroni O, Kim JK, Kim YB, Boss O (2001) Adiposeselective targeting of the GLUT4 gene impairs insulin action in muscle and liver. Nature 409:729-733

8. Klaman LD, Boss O, Peroni OD et al (2000) Increased energy expenditure, decreased adiposity, and tissue-specific insulin sensitivity in protein-tyrosine phosphatase 1B-deficient mice. Mol Cell Biol 20:5479-5489

9. Arya G, Niven D (2009) Production of haemolysins by strains of the Actinobacillus minor. Vet Microbiol 141:332-341

10. Wang ZV, Deng Y, Wang QA, Sun K, Scherer PE (2010) Identification and characterization of a promoter cassette conferring adipocyte-specific gene expression. Endocrinology 151:2933-2939

11. Kobayashi K, Inoguchi T (2005) Adipokines: therapeutic targets for metabolic syndrome. Curr Drug Targets 6:525-529

12. Loh K, Deng H, Fukushima A et al (2009) Reactive oxygen species enhance insulin sensitivity. Cell Metab 10:260-272

13. Mur C, Valverde AM, Kahn CR, Benito M (2002) Increased insulin sensitivity in IGF-I receptor-deficient brown adipocytes. Diabetes $51: 743-754$
14. Cnop M, Havel P, Utzschneider K et al (2003) Relationship of adiponectin to body fat distribution, insulin sensitivity and plasma lipoproteins: evidence for independent roles of age and sex. Diabetologia 46:459-469

15. Misso ML, Murata Y, Boon WC, Jones MEE, Britt KL, Simpson ER (2003) Cellular and molecular characterization of the adipose phenotype of the aromatase-deficient mouse. Endocrinology 144:1474-1480

16. Agouni A, Mody N, Owen C et al (2011) Liver-specific deletion of protein tyrosine phosphatase (PTP) 1B improves obesity and pharmacologically-induced endoplasmic reticulum stress. Biochem J 438:369-378

17. Delibegovic M, Zimmer D, Kauffman C et al (2009) Liver-specific deletion of protein-tyrosine phosphatase 1B (PTP1B) improves metabolic syndrome and attenuates diet-induced endoplasmic reticulum stress. Diabetes 58:590-599

18. Bence K, Delibegovic M, Xue B et al (2006) Neuronal PTP1B regulates body weight, adiposity and leptin action. Nat Med 12:917-924

19. Schuler M, Dierich A, Chambon P, Metzger D (2004) Efficient temporally controlled targeted somatic mutagenesis in hepatocytes of the mouse. Genesis 39:167-172

20. Almind K, Kahn CR (2004) Genetic determinants of energy expenditure and insulin resistance in diet-induced obesity in mice. Diabetes 53:3274-3285

21. Katsuki A, Sumida Y, Gabazza EC et al (2001) Homeostasis model assessment is a reliable indicator of insulin resistance during followup of patients with type 2 diabetes. Diabetes Care 24:362-365

22. Delibegovic M, Bence KK, Mody N et al (2007) Improved glucose homeostasis in mice with muscle-specific deletion of proteintyrosine phosphatase 1B. Mol Cell Biol 27:7727-7734

23. Bence KK (2010) Hepatic PTP1B deficiency: the promise of a treatment for metabolic syndrome? J Clin Metab Diabetes 1:27-33

24. Gum RJ, Gaede LL, Koterski SL et al (2003) Reduction of protein tyrosine phosphatase $1 \mathrm{~B}$ increases insulin-dependent signaling in ob/ob mice. Diabetes 52:21-28

25. Yip SC, Saha S, Chernoff J (2010) PTP1B: a double agent in metabolism and oncogenesis. Trends Biochem Sci 35:442-449

26. Revuelta-Cervantes J, Mayoral R, Miranda S et al (2011) Protein tyrosine phosphatase 1B (PTP1B) deficiency accelerates hepatic regeneration in mice. Am J Pathol 178:1591-1604

27. Lessard L, Stuible M, Tremblay ML (2010) The two faces of PTP1B in cancer. Biochim Biophys Acta 1804:613-619

28. Balavenkatraman KK, Aceto N, Britschgi A et al (2011) Epithelial protein-tyrosine phosphatase $1 \mathrm{~B}$ contributes to the induction of mammary tumors by HER2/Neu but is not essential for tumor maintenance. Mol Cancer Res 9:1377-1384

29. Bentires-Alj M, Neel BG (2007) Protein-tyrosine phosphatase 1B is required for HER2/Neu-induced breast cancer. Cancer Res 67:2420-2424

30. Julien SG, Dubé N, Read M et al (2007) Protein tyrosine phosphatase 1B deficiency or inhibition delays ErbB2-induced mammary tumorigenesis and protects from lung metastasis. Nat Genet 39:338-346

31. Stull AJ, Wang ZQ, Zhang XH, Yu Y, Johnson WD, Cefalu WT (2012) Skeletal muscle protein tyrosine phosphatase 1B regulates insulin sensitivity in African Americans. Diabetes 61:1415-1422

32. Kiermayer C, Conrad M, Schneider M, Schmidt J, Brielmeier M (2007) Optimization of spatiotemporal gene inactivation in mouse heart by oral application of tamoxifen citrate. Genesis 45:11-16

33. Welle S, Burgess K, Thornton CA, Tawil R (2009) Relation between extent of myostatin depletion and muscle growth in mature mice. Am J Physiol Endocrinol Metab 297:E935-E940

34. Andersson KB, Winer LH, Mørk HK, Molkentin JD, Jaisser F (2010) Tamoxifen administration routes and dosage for inducible Cre-mediated gene disruption in mouse hearts. Transgenic Res 19:715-725 
35. Lee KY, Russell SJ, Ussar S et al (2013) Lessons on conditional gene targeting in mouse adipose tissue. Diabetes 62:864-874

36. Geraghty P, Hardigan AA, Wallace AM et al (2013) The GPx1PTP1B-PP2A axis: a key determinant of airway inflammation and alveolar destruction. Am J Respir Cell Mol Biol. doi:10.1165/rcmb. 2013-0026OC

37. Shimizu S, Ugi S, Maegawa H et al (2003) Protein-tyrosine phosphatase $1 \mathrm{~B}$ as new activator for hepatic lipogenesis via sterol regulatory element-binding protein-1 gene expression. J Biol Chem 278:43095-43101

38. Saltiel AR, Kahn CR (2001) Insulin signalling and the regulation of glucose and lipid metabolism. Nature 414:799-806

39. Alonso-Chamorro M, Nieto-Vazquez I, Montori-Grau M, GomezFoix A, Fernandez-Veledo S, Lorenzo M (2011) New emerging role of protein-tyrosine phosphatase $1 \mathrm{~B}$ in the regulation of glycogen metabolism in basal and TNF- $\alpha$-induced insulin-resistant conditions in an immortalised muscle cell line isolated from mice. Diabetologia 54:1157-1168

40. Li JM, Li YC, Kong LD, Hu QH (2010) Curcumin inhibits hepatic protein-tyrosine phosphatase $1 \mathrm{~B}$ and prevents hypertriglyceridemia and hepatic steatosis in fructose-fed rats. Hepatology 51:15551566

41. Gavrilova O, Haluzik M, Matsusue K et al (2003) Liver peroxisome proliferator-activated receptor $\gamma$ contributes to hepatic steatosis, triglyceride clearance, and regulation of body fat mass. J Biol Chem 278:34268-34276

42. Banno R, Zimmer D, de Jonghe BC et al (2010) PTP1B and SHP2 in POMC neurons reciprocally regulate energy balance in mice. J Clin Invest 120:720-734

43. Brabant G, Müller G, Horn R, Anderwald C, Roden M, Nave H (2005) Hepatic leptin signaling in obesity. FASEB J 19:1048-1050

44. Brabant G, Nave H, Horn R, Anderwald C, Müller G, Roden M (2004) In vivo and in vitro evidence for a hepatic modulation of the leptin signal in rats. Eur J Clin Invest 34:831-837

45. Cohen P, Yang G, Yu X et al (2005) Induction of leptin receptor expression in the liver by leptin and food deprivation. J Biol Chem 280:10034-10039

46. Lam N, Covey S, Lewis J et al (2006) Leptin resistance following over-expression of protein tyrosine phosphatase 1B in liver. J Mol Endocrinol 36:163-174 